\title{
A SIMPLE DEFORMATION OF QUASICONFORMAL HARMONIC MAPPINGS IN THE UNIT DISK
}

\section{Dariusz Partyka and Ken-ichi Sakan}

The John Paul II Catholic University of Lublin, Faculty of Mathematics and Natural Sciences

Al. Racławickie 14, P.O. Box 129, 20-950 Lublin, Poland, and

The State School of Higher Education in Chełm, Institute of Mathematics and Information Technology, Pocztowa 54, 22-100 Chełm, Poland; partyka@kul.lublin.pl

Osaka City University, Graduate School of Science, Department of Mathematics Sugimoto, Sumiyoshi-ku, Osaka, 558, Japan; ksakan@sci.osaka-cu.ac.jp

Dedicated to Professor Bogdan Bojarski on the occasion of his eightieth birthday.

\begin{abstract}
Given a sense-preserving injective harmonic mapping $F$ in the unit disk $\mathbf{D}$ and $a \in$ C we consider a simple deformation $\mathbf{C} \ni a \mapsto F_{a}:=H+a \bar{G}$ of $F$, where $H$ and $G$ are holomorphic mappings in $\mathbf{D}$ determined by $F=H+\bar{G}$ and $G(0)=0$. We introduce a natural generalization of convexity called $\alpha$-convexity. Then we study the bi-Lipschitz behaviour of mappings $F_{a}$ under the assumption that $F$ is a quasiconformal harmonic mapping of $\mathbf{D}$ onto an $\alpha$-convex domain $F(\mathbf{D})$. As an application we show that if $F$ is a quasiconformal harmonic self-mapping of $\mathbf{D}$, then $H$ is a bi-Lipschitz mapping. Consequently, a sense-preserving harmonic self-mapping $F$ of $\mathbf{D}$ is quasiconformal iff $H$ is Lipschitz with the Jacobian of $F$ separated from zero by a positive constant in $\mathbf{D}$.
\end{abstract}

\section{Introduction}

Set $\mathbf{D}(R):=\{z \in \mathbf{C}:|z|<R\}$ for every $R>0$. Throughout the paper we always assume that $F$ is a sense-preserving injective harmonic mapping of the unit $\operatorname{disk} \mathbf{D}:=\mathbf{D}(1)$ onto a domain $\Omega$ in $\mathbf{C}$. Then $F$ is represented uniquely by

$$
F(z)=H(z)+\overline{G(z)}, \quad z \in \mathbf{D},
$$

where $H$ and $G$ are holomorphic mappings in $\mathbf{D}$ and $G(0)=0$. Moreover, from the classical Lewy's theorem it follows that the Jacobian $\mathrm{J}[F]$ does not vanish on $\mathbf{D}$; cf. [7]. Since $F$ is sense-preserving,

$$
\left|H^{\prime}(z)\right|^{2}-\left|G^{\prime}(z)\right|^{2}=|\partial F(z)|^{2}-|\bar{\partial} F(z)|^{2}=\mathrm{J}[F](z)>0, \quad z \in \mathbf{D},
$$

where $\partial:=\frac{1}{2}\left(\partial_{x}-\mathrm{i} \partial_{y}\right)$ and $\bar{\partial}:=\frac{1}{2}\left(\partial_{x}+\mathrm{i} \partial_{y}\right)$ are the so-called formal derivatives operators. Therefore the complex dilatation

$$
\mu_{F}(z):=\frac{\bar{\partial} F(z)}{\partial F(z)}=\frac{\overline{G^{\prime}(z)}}{H^{\prime}(z)}, \quad z \in \mathbf{D},
$$

doi:10.5186/aasfm.2012.3731

2010 Mathematics Subject Classification: Primary 30C55, 30C62.

Key words: Harmonic mappings, Lipschitz condition, bi-Lipchitz condition, co-Lipchitz condition, Jacobian, quasiconformal mappings.

The research of the second named author was supported by Grants-in-Aid for Scientific Research No. 22340025 and No. 20340030, Japan Society for the Promotion of Science. 
is well defined and

$$
\left\|\mu_{F}\right\|_{R, \infty}:=\operatorname{essip}_{z \in \mathbf{D}(R)}\left|\mu_{F}(z)\right|=\sup _{z \in \mathbf{D}(R)}\left|\mu_{F}(z)\right| \leq 1, \quad 0<R \leq 1 .
$$

Since $G^{\prime} / H^{\prime}$ is a holomorphic mapping, we conclude from the maximum principle that

$$
\left\|\mu_{F}\right\|_{R, \infty}<1, \quad 0<R<1 .
$$

Recently Kalaj studied the following transformation

$$
\mathbf{C} \ni a \mapsto F_{a}:=H+a \bar{G}
$$

cf. [5]. He proved that:

Theorem A. [5, Thm. 2.1] Suppose $F(\mathbf{D})$ is a convex domain in $\mathbf{C}$ and $a \in \mathbf{C}$. If $|a| \leq 1$, then $F_{a}$ is an injective close-to-convex harmonic mapping. Furthermore, if $|a|<1$, then the mapping $F_{a}$ is $(1+|a|) /(1-|a|)$-quasiconformal.

Since $H=F_{0}$, Kalaj's result shows that the transformation (0.6) provides a simple harmonic deformation of the conformal close-to-convex mapping $H$ which leads to $F$ via close-to-convex harmonic mappings $F_{a}$. Therefore the mapping

$$
\mathbf{D} \times H(\mathbf{D}) \ni(t, z) \mapsto F(t, z):=F_{t} \circ H^{-1}(z)=z+t \bar{G} \circ H^{-1}(z)
$$

is a holomorphic motion of the set $H(\mathbf{D})$, because:

(i) $F(0, z)=z$ for $z \in H(\mathbf{D})$;

(ii) $\mathbf{D} \ni t \mapsto F(t, z)$ is a holomorphic function for each $z \in H(\mathbf{D})$;

(iii) $H(\mathbf{D}) \ni z \mapsto F(t, z)$ is an injective mapping for each $t \in \mathbf{D}$.

This points out a possibility of usage of the theory of holomorphic motions for studying harmonic mappings. On the other hand side, Clunie and Sheil-Small considered in [3], similarly to (0.6), a holomorphic deformation $\mathbf{C} \ni a \mapsto H+a G$.

The above facts have motivated us to study the bi-Lipschitz property of the transformation (0.6). We start with the following observation.

Proposition 0.1. For every $L \geq 0$, if

$$
|F(w)-F(z)| \leq L|w-z|, \quad w, z \in \mathbf{D},
$$

then for every $a \in \mathbf{C}$,

$$
\left|F_{a}(w)-F_{a}(z)\right| \leq\left(1+|a|\left\|\mu_{F}\right\|_{1, \infty}\right) L|w-z|, \quad w, z \in \mathbf{D} .
$$

Proof. Since $F$ is differentiable at an arbitrarily fixed point $\zeta_{0} \in \mathbf{D}$, we have

$$
F(\zeta)-F\left(\zeta_{0}\right)=\partial F\left(\zeta_{0}\right)\left(\zeta-\zeta_{0}\right)+\bar{\partial} F\left(\zeta_{0}\right) \overline{\left(\zeta-\zeta_{0}\right)}+\mathrm{o}\left(\zeta-\zeta_{0}\right), \quad \zeta \in \mathbf{D} .
$$

Then for each $\theta \in \mathbf{R}$, we conclude from (0.8) that

$$
\begin{aligned}
\left|\partial F\left(\zeta_{0}\right) \mathrm{e}^{\mathrm{i} \theta}+\bar{\partial} F\left(\zeta_{0}\right) \mathrm{e}^{-\mathrm{i} \theta}\right| & =\frac{1}{r}\left|F\left(r \mathrm{e}^{\mathrm{i} \theta}+\zeta_{0}\right)-F\left(\zeta_{0}\right)\right|+\frac{1}{r}\left|\mathrm{o}\left(r \mathrm{e}^{\mathrm{i} \theta}\right)\right| \\
& \leq L+\frac{1}{r}\left|\mathrm{o}\left(r \mathrm{e}^{\mathrm{i} \theta}\right)\right| \rightarrow L \quad \text { as } r \rightarrow 0 .
\end{aligned}
$$

On the other hand side $\partial F\left(\zeta_{0}\right)=\left|\partial F\left(\zeta_{0}\right)\right| \mathrm{e}^{\mathrm{i} \alpha}$ and $\bar{\partial} F\left(\zeta_{0}\right)=\left|\bar{\partial} F\left(\zeta_{0}\right)\right| \mathrm{e}^{\mathrm{i} \beta}$ for some $\alpha, \beta \in \mathbf{R}$. Thus for $\theta:=(\beta-\alpha) / 2$ we obtain

$$
\left|\partial F\left(\zeta_{0}\right)\right|+\left|\bar{\partial} F\left(\zeta_{0}\right)\right| \leq L, \quad \zeta_{0} \in \mathbf{D}
$$


Given $z, w \in \mathbf{D}, z \neq w$, we define $[0 ; 1] \ni s \mapsto \gamma(s):=z+s(w-z)$. Since $\gamma([0 ; 1])$ is a line segment laying in $\mathbf{D}$ we conclude from (0.4) and (0.12) that for any $a \in \mathbf{C}$,

$$
\begin{aligned}
\frac{\left|F_{a}(w)-F_{a}(z)\right|}{|w-z|} & =\frac{1}{|w-z|}\left|\int_{0}^{1} \frac{\mathrm{d} F_{a}(\gamma(s))}{\mathrm{d} s} \mathrm{~d} s\right| \leq \int_{0}^{1}\left(\left|\partial F_{a}(\gamma(s))\right|+\mid \bar{\partial} F_{a}(\gamma(s) \mid) \mathrm{d} s\right. \\
& \leq \int_{0}^{1}\left|\partial F_{a}(\gamma(s))\right|\left(1+|a|\left\|\mu_{F}\right\|_{1, \infty}\right) \mathrm{d} s=\left(1+|a|\left\|\mu_{F}\right\|_{1, \infty}\right) L
\end{aligned}
$$

which yields (0.9).

According to Proposition 0.1 the transformation (0.6) preserves the Lipschitz property of the mapping $F$. In this paper we say that a mapping is co-Lipschitz provided it is injective and its inverse mapping is Lipschitz. In what follows we study under what conditions the mapping $F_{a}$ is co-Lipschitz provided so is the mapping $F$. This is a more sophisticated task. In Section 3 we show Theorem 3.4 which asserts that $F_{a}$ is co-Lipschitz for every $a$ in a definite disk provided $\Omega$ is an $\alpha$-convex domain with $\alpha \in[0 ; 1)$ (cf. Section 1 ) and $F$ is co-Lipschitz and quasiconformal with $\left\|\mu_{F}\right\|_{1, \infty}<\cos \frac{\alpha \pi}{2}$.

It is worth noting here the paper [2] by Chuaqui and Hernández. They studied the relationship between the injectivity of the mappings $F$ and $H$ under the assumption that $\Omega$ is a linearly connected domain. Corollary 2.2 can be treated as a counterpart of [2, Thm. 2]. Theorem 3.4 looks also quite related to [2, Thm. 3]. We would like to express our sincere thanks to the referee who pointed out the paper [2] and gave us the suggestion to improve the original Corollary 2.2 into the present one.

In the paper [12] Pavlovic showed that in the case where $F(\mathbf{D})=\mathbf{D}, F$ is quasiconformal iff $F$ is bi-Lipschitz. As an application of Theorem 4.1 we show that $H$ is a bi-Lipschitz mapping provided $F$ is quasiconformal. In consequence, we obtain another necessary and sufficient condition for quasiconformality of $F$. Namely, if $F(\mathbf{D})=\mathbf{D}$, then $F$ is quasiconformal iff $H$ is Lipschitz with the Jacobian $\mathrm{J}[F]$ of $F$ separated from zero by a positive constant in $\mathbf{D}$; cf. Corollary 4.4.

\section{Bounded turning property}

We start with the following deviation measure of a regular arc from the line segments with the same endpoints.

Definition 1.1. Given $\alpha \geq 0$ we call a continuously differentiable function $\gamma:[0 ; 1] \rightarrow \mathbf{C}$ an $\alpha$-bounded turning arc $(\alpha$-bta for short) provided there exist a continuous function $\theta:[0 ; 1] \rightarrow \mathbf{R}$ and $\theta_{0} \in \mathbf{R}$ satisfying the following properties

$$
\begin{aligned}
\gamma^{\prime}(s) & =\exp (\mathrm{i} \theta(s))\left|\gamma^{\prime}(s)\right| \neq 0, \quad 0 \leq s \leq 1, \\
\left|\theta(s)-\theta_{0}\right| & \leq \frac{\alpha \pi}{2}, \quad 0 \leq s \leq 1, \\
\gamma(1)-\gamma(0) & =|\gamma(1)-\gamma(0)| \exp \left(\mathrm{i} \theta_{0}\right) .
\end{aligned}
$$

Note that every 0 -bta $\gamma$ is a parameterization of a line segment in C, i.e. $\gamma([0 ; 1])$ is a line segment in $\mathbf{C}$. Using the bounded turning arcs we introduce the following generalization of a convex set in the complex plane.

Definition 1.2. Given $\alpha \geq 0$ a set $\Omega \subset \mathbf{C}$ is said to be $\alpha$-convex if for all $z_{1}, z_{2} \in \Omega, z_{1} \neq z_{2}$, there exists an $\alpha$-bta $\gamma$ such that $\gamma(0)=z_{1}, \gamma(1)=z_{2}$ and $\gamma([0 ; 1]) \subset \Omega$. 
Note that a 0 -convex set $\Omega \subset \mathbf{C}$ is a convex set. Therefore the parameter $\alpha$ measures the deviation of a set $\Omega$ from the convexity.

Lemma 1.3. For every $\alpha \in[0 ; 1)$ each $\alpha$-bta $\gamma$ is a rectifiable arc and its length $|\gamma|_{1}$ satisfies the following inequalities

$$
|\gamma|_{1} \cos \frac{\alpha \pi}{2} \leq|\gamma(1)-\gamma(0)| \leq|\gamma|_{1}
$$

Proof. Fix $\alpha \in[0 ; 1)$ and an $\alpha$-bta $\gamma$ with endpoints $z_{1}:=\gamma(0)$ and $z_{2}:=\gamma(1)$. By Definition 1.1 there exist a continuous function $\theta:[0 ; 1] \rightarrow \mathbf{R}$ and $\theta_{0} \in \mathbf{R}$ satisfying the properties (1.1), (1.2) and (1.3). Then from (1.3) it follows that

$$
\left|z_{2}-z_{1}\right|=|\gamma(1)-\gamma(0)|=(\gamma(1)-\gamma(0)) \mathrm{e}^{-\mathrm{i} \theta_{0}} .
$$

Hence and by (1.1) and (1.2) we have

$$
\begin{aligned}
\left|z_{2}-z_{1}\right| & =\operatorname{Re}\left((\gamma(1)-\gamma(0)) \mathrm{e}^{-\mathrm{i} \theta_{0}}\right)=\operatorname{Re} \int_{0}^{1} \gamma^{\prime}(s) \mathrm{e}^{-\mathrm{i} \theta_{0}} \mathrm{~d} s \\
& =\int_{0}^{1}\left|\gamma^{\prime}(s)\right| \operatorname{Re} \mathrm{e}^{\mathrm{i}\left(\theta(s)-\theta_{0}\right)} \mathrm{d} s=\int_{0}^{1}\left|\gamma^{\prime}(s)\right| \cos \left(\theta(s)-\theta_{0}\right) \mathrm{d} s \\
& \geq \int_{0}^{1}\left|\gamma^{\prime}(s)\right| \cos \frac{\alpha \pi}{2} \mathrm{~d} s=\cos \frac{\alpha \pi}{2} \int_{0}^{1}\left|\gamma^{\prime}(s)\right| \mathrm{d} s=|\gamma|{ }_{1} \cos \frac{\alpha \pi}{2},
\end{aligned}
$$

which proves the first inequality in (1.4). On the other hand side

$$
\left|z_{2}-z_{1}\right|=\left|\int_{0}^{1} \gamma^{\prime}(s) \mathrm{d} s\right| \leq \int_{0}^{1}\left|\gamma^{\prime}(s)\right| \mathrm{d} s=|\gamma|_{1}
$$

which shows the second inequality in (1.4).

\section{Auxiliary properties of harmonic mappings}

In this section we study the mappings $H$ and $G$ associated with the mapping $F$ by the equality (0.1).

Lemma 2.1. Given $\alpha \in[0 ; 1)$ suppose that $\Omega:=F(\mathbf{D})$ is an $\alpha$-convex domain. Then for every pair of $z_{1}, z_{2} \in \mathbf{D}, z_{1} \neq z_{2}$, there exists $R \in(0 ; 1)$ such that

$$
-\frac{1}{\cos \frac{\alpha \pi}{2}} \cdot \frac{k}{1-k} \leq \operatorname{Re} \frac{\bar{G}\left(z_{2}\right)-\bar{G}\left(z_{1}\right)}{F\left(z_{2}\right)-F\left(z_{1}\right)} \leq \frac{1-\cos \frac{\alpha \pi}{2}}{\cos \frac{\alpha \pi}{2}} \cdot \frac{k}{1-k^{2}}+\frac{k}{1+k}
$$

as well as

$$
\frac{1}{1+k}-\frac{1-\cos \frac{\alpha \pi}{2}}{\cos \frac{\alpha \pi}{2}} \cdot \frac{k}{1-k^{2}} \leq \operatorname{Re} \frac{H\left(z_{2}\right)-H\left(z_{1}\right)}{F\left(z_{2}\right)-F\left(z_{1}\right)} \leq 1+\frac{1}{\cos \frac{\alpha \pi}{2}} \cdot \frac{k}{1-k}
$$

for every $k$ satisfying $\left\|\mu_{F}\right\|_{R, \infty} \leq k<1$. Moreover, the following inequalities hold

$$
\begin{aligned}
\left|\operatorname{Im} \frac{H\left(z_{2}\right)-H\left(z_{1}\right)}{F\left(z_{2}\right)-F\left(z_{1}\right)}\right| & =\left|\operatorname{Im} \frac{\bar{G}\left(z_{2}\right)-\bar{G}\left(z_{1}\right)}{F\left(z_{2}\right)-F\left(z_{1}\right)}\right| \leq \frac{k+k^{2} \sin \frac{\alpha \pi}{2}}{\left(1-k^{2}\right) \cos \frac{\alpha \pi}{2}}, \\
\left|\frac{G\left(z_{2}\right)-G\left(z_{1}\right)}{F\left(z_{2}\right)-F\left(z_{1}\right)}\right| & \leq \frac{k}{(1-k) \cos \frac{\alpha \pi}{2}}, \\
\left|\frac{H\left(z_{2}\right)-H\left(z_{1}\right)}{F\left(z_{2}\right)-F\left(z_{1}\right)}\right| & \leq \frac{1}{(1-k) \cos \frac{\alpha \pi}{2}} .
\end{aligned}
$$


Proof. Take arbitrary points $z_{1}, z_{2} \in \mathbf{D}$ such that $z_{1} \neq z_{2}$. Since $\Omega$ is an $\alpha$ convex domain, there exists an $\alpha$-bta $\gamma$ such that $\gamma(0)=F\left(z_{1}\right), \gamma(1)=F\left(z_{2}\right)$ and $\gamma([0 ; 1]) \subset \Omega$. By Definition 1.1 there exist a continuous function $\theta:[0 ; 1] \rightarrow \mathbf{R}$ and $\theta_{0} \in \mathbf{R}$ satisfying the properties (1.1), (1.2) and (1.3). Then $\sigma:=F^{-1} \circ \gamma$ is an arc in $\mathbf{D}$ joining $z_{1}$ with $z_{2}$. Since $\sigma([0 ; 1])$ is a compact subset of $\mathbf{D}, \sigma([0 ; 1]) \subset \mathbf{D}(R)$ for certain $R \in(0 ; 1)$. Hence and by $(0.5)$,

$$
\left|\mu_{F}(\sigma(s))\right| \leq\left\|\mu_{F}\right\|_{R, \infty}<1, \quad 0 \leq s \leq 1 .
$$

Applying Lemma 1.3 we also have

$$
|\gamma|_{1} \cos \frac{\alpha \pi}{2} \leq\left|F\left(z_{2}\right)-F\left(z_{1}\right)\right| \leq|\gamma|_{1} .
$$

Since for every $z \in \mathbf{D}$,

$$
\begin{aligned}
& 1=\partial\left(F^{-1} \circ F\right)(z)=\partial F^{-1}(F(z)) H^{\prime}(z)+\bar{\partial} F^{-1}(F(z)) G^{\prime}(z) \\
& 0=\bar{\partial}\left(F^{-1} \circ F\right)(z)=\partial F^{-1}(F(z)) \overline{G^{\prime}(z)}+\bar{\partial} F^{-1}(F(z)) \overline{H^{\prime}(z),}
\end{aligned}
$$

we easily see that

$$
\begin{aligned}
& \partial F^{-1}(F(z))=\frac{\bar{\partial} \bar{F}}{J[F](z)}=\frac{\overline{H^{\prime}(z)}}{\left|H^{\prime}(z)\right|^{2}-\left|G^{\prime}(z)\right|^{2}} \\
& \bar{\partial} F^{-1}(F(z))=-\frac{\bar{\partial} F}{\mathrm{~J}[F](z)}=-\frac{\overline{G^{\prime}(z)}}{\left|H^{\prime}(z)\right|^{2}-\left|G^{\prime}(z)\right|^{2}} .
\end{aligned}
$$

Using now (1.1) and (2.8) we obtain

$$
\begin{aligned}
& G\left(z_{2}\right)-G\left(z_{1}\right)=\int_{\sigma} G^{\prime}(z) \mathrm{d} z=\int_{0}^{1} G^{\prime}(\sigma(s)) \frac{\mathrm{d}}{\mathrm{d} s} \sigma(s) \mathrm{d} s \\
& =\int_{0}^{1} G^{\prime}(\sigma(s))\left[\partial F^{-1}(\gamma(s)) \gamma^{\prime}(s)+\bar{\partial} F^{-1}(\gamma(s)) \overline{\gamma^{\prime}(s)}\right] \mathrm{d} s \\
& =\int_{0}^{1} G^{\prime}(\sigma(s))\left[\partial F^{-1}(F(\sigma(s))) \gamma^{\prime}(s)+\bar{\partial} F^{-1}(F(\sigma(s))) \overline{\gamma^{\prime}(s)}\right] \mathrm{d} s \\
& =\int_{0}^{1} G^{\prime}(\sigma(s))\left[\frac{\overline{H^{\prime}(\sigma(s))}}{\mathrm{J}[F](\sigma(s))} \mathrm{e}^{\mathrm{i} \theta(s)}-\frac{\overline{G^{\prime}(\sigma(s))}}{\mathrm{J}[F](\sigma(s))} \mathrm{e}^{-\mathrm{i} \theta(s)}\right]\left|\gamma^{\prime}(s)\right| \mathrm{d} s \\
& =\int_{0}^{1} \frac{G^{\prime}(\sigma(s)) \overline{H^{\prime}(\sigma(s))} \mathrm{e}^{\mathrm{i} \theta(s)}-\left|G^{\prime}(\sigma(s))\right|^{2} \mathrm{e}^{-\mathrm{i} \theta(s)}}{\left|H^{\prime}(\sigma(s))\right|^{2}-\left|G^{\prime}(\sigma(s))\right|^{2}} \cdot\left|\gamma^{\prime}(s)\right| \mathrm{d} s \\
& =\int_{0}^{1} \frac{\mu_{F}(\sigma(s)) \frac{G^{\prime}(\sigma(s))}{G^{\prime}(\sigma(s))} \mathrm{e}^{\mathrm{i} \theta(s)}-\left|\mu_{F}(\sigma(s))\right|^{2} \mathrm{e}^{-\mathrm{i} \theta(s)}}{1-\left|\mu_{F}(\sigma(s))\right|^{2}} \cdot\left|\gamma^{\prime}(s)\right| \mathrm{d} s .
\end{aligned}
$$

Hence

$$
\begin{aligned}
& \mathrm{e}^{-\mathrm{i} \theta_{0}} \overline{\left(G\left(z_{2}\right)-G\left(z_{1}\right)\right)} \\
& =\int_{0}^{1} \frac{\overline{\mu_{F}(\sigma(s))} \frac{\overline{G^{\prime}(\sigma(s))}}{G^{\prime}(\sigma(s))} \mathrm{e}^{-\mathrm{i}\left(\theta_{0}+\theta(s)\right)}-\left|\mu_{F}(\sigma(s))\right|^{2} \mathrm{e}^{\mathrm{i}\left(\theta(s)-\theta_{0}\right)}}{1-\left|\mu_{F}(\sigma(s))\right|^{2}} \cdot\left|\gamma^{\prime}(s)\right| \mathrm{d} s .
\end{aligned}
$$

By (1.2), we obtain

$$
\operatorname{Re} \mathrm{e}^{\mathrm{i}\left(\theta_{0}-\theta(s)\right)} \geq \cos \frac{\alpha \pi}{2}>0, \quad 0 \leq s \leq 1
$$


Combining this with (2.9), (2.6) and (2.7) we get

$$
\begin{aligned}
\operatorname{Re}\left[\mathrm{e}^{-\mathrm{i} \theta_{0}}\right. & \left.\overline{\left(G\left(z_{2}\right)-G\left(z_{1}\right)\right)}\right] \\
\leq & \int_{0}^{1} \frac{\mid \overline{\mu_{F}(\sigma(s))} \frac{G^{\prime}(\sigma(s))}{G^{\prime}(\sigma(s))}}{\left.\mathrm{e}^{-\mathrm{i}\left(\theta_{0}+\theta(s)\right)}|-| \mu_{F}(\sigma(s))\right|^{2} \cos \frac{\alpha \pi}{2}} \cdot\left|\gamma^{\prime}(s)\right| \mathrm{d} s \\
= & \int_{0}^{1} \frac{\left(1-\cos \frac{\alpha \pi}{2}\right)\left|\mu_{F}(\sigma(s))\right|}{1-\left|\mu_{F}(\sigma(s))\right|^{2}} \cdot\left|\gamma^{\prime}(s)\right| \mathrm{d} s \\
& +\int_{0}^{1} \frac{\left(\left|\mu_{F}(\sigma(s))\right|-\left|\mu_{F}(\sigma(s))\right|^{2}\right) \cos \frac{\alpha \pi}{2}}{1-\left|\mu_{F}(\sigma(s))\right|^{2}} \cdot\left|\gamma^{\prime}(s)\right| \mathrm{d} s \\
\leq & |\gamma|_{1}\left(\left(1-\cos \frac{\alpha \pi}{2}\right) \cdot \frac{\left\|\mu_{F}\right\|_{R, \infty}}{1-\left\|\mu_{F}\right\|_{R, \infty}^{2}}+\cos \frac{\alpha \pi}{2} \cdot \frac{\left\|\mu_{F}\right\|_{R, \infty}}{1+\left\|\mu_{F}\right\|_{R, \infty}}\right) \\
\leq & \left|F\left(z_{2}\right)-F\left(z_{1}\right)\right|\left(\frac{1-\cos \frac{\alpha \pi}{2}}{\cos \frac{\alpha \pi}{2}} \cdot \frac{\left\|\mu_{F}\right\|_{R, \infty}}{1-\left\|\mu_{F}\right\|_{R, \infty}^{2}}+\frac{\left\|\mu_{F}\right\|_{R, \infty}}{1+\left\|\mu_{F}\right\|_{R, \infty}}\right)
\end{aligned}
$$

which together with (1.3) yields the second inequality in (2.1). On the other hand side, we conclude from (2.9), (2.6) and (2.7) that

$$
\begin{aligned}
\operatorname{Re}\left[\mathrm{e}^{-\mathrm{i} \theta_{0}} \overline{\left(G\left(z_{2}\right)-G\left(z_{1}\right)\right)}\right] & \geq \int_{0}^{1} \frac{-\left|\overline{\mu_{F}(\sigma(s))}\right|-\left|\mu_{F}(\sigma(s))\right|^{2}}{1-\left|\mu_{F}(\sigma(s))\right|^{2}} \cdot\left|\gamma^{\prime}(s)\right| \mathrm{d} s \\
& =-\int_{0}^{1} \frac{\left|\overline{\mu_{F}(\sigma(s))}\right|}{1-\left|\mu_{F}(\sigma(s))\right|} \cdot\left|\gamma^{\prime}(s)\right| \mathrm{d} s \geq-|\gamma|_{1} \frac{\left\|\mu_{F}\right\|_{R, \infty}}{1-\left\|\mu_{F}\right\|_{R, \infty}} \\
& \geq-\frac{\left|F\left(z_{2}\right)-F\left(z_{1}\right)\right|}{\cos \frac{\alpha \pi}{2}} \cdot \frac{\left\|\mu_{F}\right\|_{R, \infty}}{1-\left\|\mu_{F}\right\|_{R, \infty}},
\end{aligned}
$$

which together with (1.3) yields the first inequality in (2.1). hence

From (0.1) it follows that $F\left(z_{2}\right)-F\left(z_{1}\right)=H\left(z_{2}\right)-H\left(z_{1}\right)+\bar{G}\left(z_{2}\right)-\bar{G}\left(z_{1}\right)$, and

$$
\begin{aligned}
& \operatorname{Re} \frac{H\left(z_{2}\right)-H\left(z_{1}\right)}{F\left(z_{2}\right)-F\left(z_{1}\right)}=1-\operatorname{Re} \frac{\bar{G}\left(z_{2}\right)-\bar{G}\left(z_{1}\right)}{F\left(z_{2}\right)-F\left(z_{1}\right)} \\
& \operatorname{Im} \frac{H\left(z_{2}\right)-H\left(z_{1}\right)}{F\left(z_{2}\right)-F\left(z_{1}\right)}=-\operatorname{Im} \frac{\bar{G}\left(z_{2}\right)-\bar{G}\left(z_{1}\right)}{F\left(z_{2}\right)-F\left(z_{1}\right)}
\end{aligned}
$$

This together with (2.1) yields the inequalities (2.2) and the equality in (2.3). From (2.9) we conclude that

$$
\begin{aligned}
& \operatorname{Im}\left[\mathrm{e}^{-\mathrm{i} \theta_{0}} \overline{\left(G\left(z_{2}\right)-G\left(z_{1}\right)\right)}\right] \\
& =\int_{0}^{1} \frac{\operatorname{Im}\left[\overline{\mu_{F}(\sigma(s))} \frac{\overline{G^{\prime}(\sigma(s))}}{G^{\prime}(\sigma(s))} \mathrm{e}^{-\mathrm{i}\left(\theta_{0}+\theta(s)\right)}\right]-\left|\mu_{F}(\sigma(s))\right|^{2} \operatorname{Im} \mathrm{e}^{\mathrm{i}\left(\theta(s)-\theta_{0}\right)}}{1-\left|\mu_{F}(\sigma(s))\right|^{2}} \cdot\left|\gamma^{\prime}(s)\right| \mathrm{d} s .
\end{aligned}
$$

By (1.2) we have

$$
\left|\operatorname{Im} \mathrm{e}^{\mathrm{i}\left(\theta_{0}-\theta(s)\right)}\right| \leq \sin \frac{\alpha \pi}{2}, \quad 0 \leq s \leq 1
$$


Thus applying (2.6) and (2.7) we get

$$
\begin{aligned}
& \left|\operatorname{Im}\left[\mathrm{e}^{-\mathrm{i} \theta_{0}} \overline{\left(G\left(z_{2}\right)-G\left(z_{1}\right)\right)}\right]\right| \\
& \leq \int_{0}^{1} \frac{\left|\mu_{F}(\sigma(s))\right|+\left|\mu_{F}(\sigma(s))\right|^{2}\left|\operatorname{Im} \mathrm{e}^{\mathrm{i}\left(\theta(s)-\theta_{0}\right)}\right|}{1-\left|\mu_{F}(\sigma(s))\right|^{2}} \cdot\left|\gamma^{\prime}(s)\right| \mathrm{d} s \\
& \leq\left.|\gamma|\right|_{1} \frac{k+k^{2} \sin \frac{\alpha \pi}{2}}{1-k^{2}} \leq\left|F\left(z_{2}\right)-F\left(z_{1}\right)\right| \frac{k+k^{2} \sin \frac{\alpha \pi}{2}}{\left(1-k^{2}\right) \cos \frac{\alpha \pi}{2}},
\end{aligned}
$$

which together with (1.3) yields the inequality in (2.3). Applying (2.9) once again we see that

$$
\begin{aligned}
& \left|\mathrm{e}^{-\mathrm{i} \theta_{0}} \overline{\left(G\left(z_{2}\right)-G\left(z_{1}\right)\right)}\right| \leq \int_{0}^{1} \frac{\left|\mu_{F}(\sigma(s))\right|+\left|\mu_{F}(\sigma(s))\right|^{2}}{1-\left|\mu_{F}(\sigma(s))\right|^{2}} \cdot\left|\gamma^{\prime}(s)\right| \mathrm{d} s \\
& \leq|\gamma|_{1} \frac{k}{1-k} \leq\left|F\left(z_{2}\right)-F\left(z_{1}\right)\right| \frac{k}{(1-k) \cos \frac{\alpha \pi}{2}},
\end{aligned}
$$

which leads to (2.4). Using (1.1) and the formulas (2.8) we have

$$
\begin{aligned}
H\left(z_{2}\right)-H\left(z_{1}\right) & =\int_{\sigma} H^{\prime}(z) \mathrm{d} z=\int_{0}^{1} H^{\prime}(\sigma(s)) \frac{\mathrm{d}}{\mathrm{d} s} \sigma(s) \mathrm{d} s \\
& =\int_{0}^{1} H^{\prime}(\sigma(s))\left[\partial F^{-1}(\gamma(s)) \gamma^{\prime}(s)+\bar{\partial} F^{-1}(\gamma(s)) \overline{\gamma^{\prime}(s)}\right] \mathrm{d} s \\
& =\int_{0}^{1} H^{\prime}(\sigma(s))\left[\partial F^{-1}(F(\sigma(s))) \gamma^{\prime}(s)+\bar{\partial} F^{-1}(F(\sigma(s))) \overline{\gamma^{\prime}(s)}\right] \mathrm{d} s \\
& =\int_{0}^{1} H^{\prime}(\sigma(s))\left[\frac{\overline{H^{\prime}(\sigma(s))}}{\mathrm{J}[F](\sigma(s))} \mathrm{e}^{\mathrm{i} \theta(s)}-\frac{\overline{G^{\prime}(\sigma(s))}}{\mathrm{J}[F](\sigma(s))} \mathrm{e}^{-\mathrm{i} \theta(s)}\right]\left|\gamma^{\prime}(s)\right| \mathrm{d} s \\
& =\int_{0}^{1} \frac{\left|H^{\prime}(\sigma(s))\right|^{2} \mathrm{e}^{\mathrm{i} \theta(s)}-H^{\prime}(\sigma(s)) \overline{G^{\prime}(\sigma(s))} \mathrm{e}^{-\mathrm{i} \theta(s)}}{\left|H^{\prime}((\sigma(s)))\right|^{2}-\left|G^{\prime}((\sigma(s)))\right|^{2}} \cdot\left|\gamma^{\prime}(s)\right| \mathrm{d} s \\
& =\int_{0}^{1} \frac{\mathrm{e}^{\mathrm{i} \theta(s)}-\overline{\mu_{F}(\sigma(s))} \frac{\bar{G}^{\prime}(\sigma(s))}{G^{\prime}(\sigma(s))}}{\mathrm{e}^{-\mathrm{i} \theta(s)}} \cdot\left|\gamma^{\prime}(s)\right| \mathrm{d} s .
\end{aligned}
$$

Hence and by (2.6) and (2.7) we see that

$$
\begin{aligned}
\left|H\left(z_{2}\right)-H\left(z_{1}\right)\right| & \leq \int_{0}^{1} \frac{1+\left|\mu_{F}(\sigma(s))\right|}{1-\left|\mu_{F}(\sigma(s))\right|^{2}} \cdot\left|\gamma^{\prime}(s)\right| \mathrm{d} s=\int_{0}^{1} \frac{\left|\gamma^{\prime}(s)\right|}{1-\left|\mu_{F}(\sigma(s))\right|} \mathrm{d} s \\
& \leq \frac{|\gamma|_{1}}{1-\left\|\mu_{F}\right\|_{R, \infty}} \leq \frac{\left|F\left(z_{2}\right)-F\left(z_{1}\right)\right|}{\cos \frac{\alpha \pi}{2}} \cdot \frac{1}{1-\left\|\mu_{F}\right\|_{R, \infty}}
\end{aligned}
$$

which leads to (2.5), and the proof is complete.

Corollary 2.2. Given $\alpha \in[0 ; 1)$ suppose that $\Omega:=F(\mathbf{D})$ is an $\alpha$-convex domain. If $\left\|\mu_{F}\right\|_{1, \infty} \leq \cos \frac{\alpha \pi}{2}$, then $H$ is an injective mapping. In particular, $H$ is an injective mapping provided $\Omega$ is a convex domain.

Proof. Take arbitrary points $z_{1}, z_{2} \in \mathbf{D}$ such that $z_{1} \neq z_{2}$. Suppose first that $\left\|\mu_{F}\right\|_{R, \infty}=\cos \frac{\alpha \pi}{2}$ for certain $R \in(0 ; 1)$. Since $G^{\prime} / H^{\prime}$ is a holomorphic mapping, we conclude from the maximum principle that $G^{\prime} / H^{\prime}$ is a constant one, and so there 
exists $c \in \mathbf{C}$ such that $G^{\prime}(z)=c H^{\prime}(z)$ as $z \in \mathbf{D}$. Hence

$$
\begin{aligned}
0 & <\left|F\left(z_{2}\right)-F\left(z_{1}\right)\right|=\left|H\left(z_{2}\right)-H\left(z_{1}\right)+\overline{\left(G\left(z_{2}\right)-G\left(z_{1}\right)\right)}\right| \\
& =\left|H\left(z_{2}\right)-H\left(z_{1}\right)+\overline{c\left(H\left(z_{2}\right)-H\left(z_{1}\right)\right)}\right| \leq(1+|c|)\left|H\left(z_{2}\right)-H\left(z_{1}\right)\right| .
\end{aligned}
$$

This implies $H\left(z_{2}\right) \neq H\left(z_{1}\right)$, and therefore $H$ is an injective mapping.

It remains to consider the opposite case, where $\left\|\mu_{F}\right\|_{R, \infty}<\cos \frac{\alpha \pi}{2}$ for all $R \in$ $(0 ; 1)$. Since $\Omega$ is an $\alpha$-convex domain and $0 \leq \alpha<1$, we conclude from Lemma 2.1 that the first inequality in $(2.2)$ holds with $k:=\left\|\mu_{F}\right\|_{R, \infty}$ for certain $R \in(0 ; 1)$, and so

$$
\begin{aligned}
\operatorname{Re} \frac{H\left(z_{2}\right)-H\left(z_{1}\right)}{F\left(z_{2}\right)-F\left(z_{1}\right)} & \geq \frac{1}{1+\left\|\mu_{F}\right\|_{R, \infty}}-\frac{1-\cos \frac{\alpha \pi}{2}}{\cos \frac{\alpha \pi}{2}} \cdot \frac{\left\|\mu_{F}\right\|_{R, \infty}}{1-\left\|\mu_{F}\right\|_{R, \infty}^{2}} \\
& =\frac{\cos \frac{\alpha \pi}{2}-\left\|\mu_{F}\right\|_{R, \infty}}{\left(1-\left\|\mu_{F}\right\|_{R, \infty}^{2}\right) \cos \frac{\alpha \pi}{2}}>0 .
\end{aligned}
$$

Hence $H\left(z_{2}\right) \neq H\left(z_{1}\right)$, and therefore $H$ is an injective mapping.

By Definition 1.2 each convex domain is a 0 -convex domain. Moreover, from (0.5) it follows that $\left\|\mu_{F}\right\|_{R, \infty}<1=\cos (0)$ for $0<R<1$. Thus, by the first part of the corollary, the mapping $H$ is injective provided $\Omega$ is a convex domain.

Remark 2.3. From Lemma 2.1 we can easily derive the following result by J. Clunie and T. Sheil-Small [3, Corollary 5.8]: If $\Omega:=F(\mathbf{D})$ is a convex domain, then

$$
\left|G\left(z_{2}\right)-G\left(z_{1}\right)\right|<\left|H\left(z_{2}\right)-H\left(z_{1}\right)\right|, \quad z_{1}, z_{2} \in \mathbf{D}, z_{1} \neq z_{2} .
$$

For the proof, fix $z_{1}, z_{2} \in \mathbf{D}$ such that $z_{1} \neq z_{2}$. Setting

$$
a:=\operatorname{Re} \frac{\bar{G}\left(z_{2}\right)-\bar{G}\left(z_{1}\right)}{F\left(z_{2}\right)-F\left(z_{1}\right)}, \quad b:=\operatorname{Re} \frac{H\left(z_{2}\right)-H\left(z_{1}\right)}{F\left(z_{2}\right)-F\left(z_{1}\right)} \quad \text { and } \quad c:=\operatorname{Im} \frac{H\left(z_{2}\right)-H\left(z_{1}\right)}{F\left(z_{2}\right)-F\left(z_{1}\right)}
$$

we deduce from (0.1) that $a+b=1$,

$$
\operatorname{Im} \frac{\bar{G}\left(z_{2}\right)-\bar{G}\left(z_{1}\right)}{F\left(z_{2}\right)-F\left(z_{1}\right)}=-c \text { and }\left|\frac{\bar{G}\left(z_{2}\right)-\bar{G}\left(z_{1}\right)}{F\left(z_{2}\right)-F\left(z_{1}\right)}\right|^{2}=(1-b)^{2}+c^{2} .
$$

Since $\Omega$ is a 0 -convex domain, we conclude from Lemma 2.1 that the first inequality in (2.2) holds with $k:=\left\|\mu_{F}\right\|_{R, \infty}$ for certain $R \in(0 ; 1)$, and so $b \geq\left(1+\left\|\mu_{F}\right\|_{R, \infty}\right)^{-1}$. By $(0.5),\left\|\mu_{F}\right\|_{R, \infty}<1=\cos (0)$. Thus $b>1 / 2$, and so $(1-\bar{b})^{2}+c^{2}<b^{2}+c^{2}$. Combining this with (2.12) and

$$
\left|\frac{H\left(z_{2}\right)-H\left(z_{1}\right)}{F\left(z_{2}\right)-F\left(z_{1}\right)}\right|^{2}=b^{2}+c^{2}
$$

we obtain (2.11).

The next lemma extends the result of Clunie and Sheil-Small.

Lemma 2.4. Given $\alpha \in[0 ; 1)$ suppose that $\Omega:=F(\mathbf{D})$ is an $\alpha$-convex domain and that $\left\|\mu_{F}\right\|_{1, \infty} \leq k<\cos \frac{\alpha \pi}{2}$. Then for all $z_{1}, z_{2} \in \mathbf{D}, z_{1} \neq z_{2}$,

$$
\left|\frac{G\left(z_{2}\right)-G\left(z_{1}\right)}{H\left(z_{2}\right)-H\left(z_{1}\right)}\right| \leq S_{1}(k, \alpha):=\max \left\{1, k \frac{1-k \cos \frac{\alpha \pi}{2}}{\cos \frac{\alpha \pi}{2}-k}\right\} .
$$


Moreover, suppose additionally that $\alpha=0$ or $k \leq \tan \frac{(1-\alpha) \pi}{4}<1$ in case $\alpha \in(0 ; 1)$. Then

$$
\left|\frac{G\left(z_{2}\right)-G\left(z_{1}\right)}{H\left(z_{2}\right)-H\left(z_{1}\right)}\right| \leq S_{2}(k, \alpha):=k \cdot \sqrt{\frac{\left(1-k \cos \frac{\alpha \pi}{2}\right)^{2}+\left(1+k \sin \frac{\alpha \pi}{2}\right)^{2}}{\left(\cos \frac{\alpha \pi}{2}-k\right)^{2}+k^{2}\left(1+k \sin \frac{\alpha \pi}{2}\right)^{2}}}
$$

Proof. Given $\alpha \in[0 ; 1)$ suppose that $\Omega:=F(\mathbf{D})$ is an $\alpha$-convex domain. Fix $z_{1}, z_{2} \in \mathbf{D}$ such that $z_{1} \neq z_{2}$. Under notations from Remark 2.3 we conclude from (2.12) and (2.13) that

$$
\left|\frac{\bar{G}\left(z_{2}\right)-\bar{G}\left(z_{1}\right)}{H\left(z_{2}\right)-H\left(z_{1}\right)}\right|^{2}=\frac{(1-b)^{2}+c^{2}}{b^{2}+c^{2}}=\frac{1-2 b}{b^{2}+c^{2}}+1 .
$$

From the assumption $\left\|\mu_{F}\right\|_{1, \infty} \leq k<\cos \frac{\alpha \pi}{2}$ and Lemma 2.1 it follows that

$$
0<\frac{1-(\lambda+1) k}{1-k^{2}}=: b_{1} \leq b \leq b_{2}:=\frac{1+\lambda k}{1-k},
$$

where $\lambda:=\left(1-\cos \frac{\alpha \pi}{2}\right) / \cos \frac{\alpha \pi}{2}$. Hence

$$
\frac{1-2 b}{b^{2}+c^{2}} \leq \max \left\{\frac{1-2 b_{1}}{b_{1}^{2}+c^{2}}, \frac{1-2 b_{2}}{b_{2}^{2}+c^{2}}\right\} .
$$

It is easy to check that

$$
\frac{1-2 b_{1}}{b_{1}^{2}+c^{2}} \geq \frac{1-2 b_{2}}{b_{2}^{2}+c^{2}} \Longleftrightarrow b_{1} b_{2}-\frac{b_{1}+b_{2}}{2} \leq c^{2}
$$

Since

$$
b_{1} b_{2}-\frac{b_{1}+b_{2}}{2}=-\frac{\lambda k^{2}(3-k+2 \lambda)}{2\left(1-k^{2}\right)(1-k)} \leq 0 \leq c^{2},
$$

we conclude from (2.18), (2.19) and (2.16) that

$$
\left|\frac{\bar{G}\left(z_{2}\right)-\bar{G}\left(z_{1}\right)}{H\left(z_{2}\right)-H\left(z_{1}\right)}\right|^{2} \leq 1+\frac{1-2 b_{1}}{b_{1}^{2}+c^{2}} .
$$

If $b_{1} \leq 1 / 2$, then

$$
1 \leq \frac{1-2 b_{1}}{b_{1}^{2}+c^{2}}+1 \leq \frac{1-2 b_{1}}{b_{1}^{2}}+1=\frac{\left(1-b_{1}\right)^{2}}{b_{1}^{2}}=\left(\frac{1}{b_{1}}-1\right)^{2} .
$$

This together with (2.20) yields the estimation (2.14), because the right hand side in (2.20) is less than 1 provided $b_{1}>1 / 2$. Then

Assume now additionally that $\alpha=0$ or $k \leq \tan \frac{(1-\alpha) \pi}{4}<1$ in case $\alpha \in(0 ; 1)$.

$$
b_{1}-\frac{1}{2}=\frac{k^{2}-2(\lambda+1) k+1}{2\left(1-k^{2}\right)}=\frac{\left(k-\tan \frac{(1-\alpha) \pi}{4}\right)\left(k-\frac{1+\sin \frac{\alpha \pi}{2}}{\cos \frac{\alpha \pi}{2}}\right)}{2\left(1-k^{2}\right)} \geq 0 .
$$

Applying now the inequality in (2.3) and the first equality in (2.12) we obtain

$$
|c| \leq \frac{k+k^{2} \sin \frac{\alpha \pi}{2}}{\left(1-k^{2}\right) \cos \frac{\alpha \pi}{2}},
$$

and consequently,

$$
1+\frac{1-2 b_{1}}{b_{1}^{2}+c^{2}} \leq S_{2}(k, \alpha)^{2}
$$


Combining this with the inequality (2.20) we obtain the estimation (2.15).

Remark 2.5. It is easily seen that the functions $S_{1}$ and $S_{2}$ defined in Lemma 2.4 have the following properties:

(i) $S_{1}(k, 0)=1$ and $S_{2}(k, 0)=k \cdot \sqrt{\frac{(1-k)^{2}+1}{(1-k)^{2}+k^{2}}}$ for $k \in[0 ; 1)$.

(ii) If $0<\alpha<1$, then

$$
S_{1}(k, \alpha):= \begin{cases}1 & \text { as } 0 \leq k \leq \tan \frac{(1-\alpha) \pi}{4} ; \\ k \frac{1-k \cos \frac{\alpha \pi}{2}}{\cos \frac{\alpha \pi}{2}-k} & \text { as } \tan \frac{(1-\alpha) \pi}{4}<k<\cos \frac{\alpha \pi}{2} .\end{cases}
$$

(iii) If $0<\alpha<1$, then the function $S_{1}(\cdot, \alpha)$ is continuous in $\left[0 ; \cos \frac{\alpha \pi}{2}\right)$, strictly increasing in $\left(\tan \frac{(1-\alpha) \pi}{4} ; \cos \frac{\alpha \pi}{2}\right)$ and $S_{1}(k, \alpha) \rightarrow+\infty$ as $k \rightarrow \cos \frac{\alpha \pi}{2}$.

(iv) The function $S_{2}(\cdot, 0)$ is strictly increasing in $[0 ; 1), S_{2}(k, 0)>k$ for $k \in(0 ; 1)$ and $S_{2}(k, 0) \rightarrow 1$ as $k \rightarrow 1$.

(v) If $0<\alpha<1$, then $k<S_{2}(k, \alpha)<1$ for $k \in\left(0\right.$; $\left.\tan \frac{(1-\alpha) \pi}{4}\right)$ and $S_{2}(k, \alpha) \rightarrow 1$ as $k \rightarrow \tan \frac{(1-\alpha) \pi}{4}$.

\section{The Lipschitz and co-Lipschitz properties of harmonic mappings}

In this section we present various results dealing with Lipschitz and co-Lipschitz properties of harmonic mappings $F_{a}:=H+a \bar{G}$, provided the mapping $F$ maps the unit disk $\mathbf{D}$ onto an $\alpha$-convex domain. Note that $F_{0}=H$. The only exception where we have no need to require that $F(\mathbf{D})$ is an $\alpha$-convex domain for any $\alpha \geq 0$, is the following lemma.

Lemma 3.1. If $F$ maps the unit disk $\mathbf{D}$ onto a simply connected domain and there exists a constant $L>0$ such that

$$
\frac{1}{L}\left|H\left(z_{2}\right)-H\left(z_{1}\right)\right| \leq\left|F\left(z_{2}\right)-F\left(z_{1}\right)\right|, \quad z_{1}, z_{2} \in \mathbf{D},
$$

then $L \geq 1$ and $F$ is a quasiconformal mapping with $\left\|\mu_{F}\right\|_{1, \infty} \leq 1-\frac{1}{L}$.

Proof. Fix $z \in \mathbf{D}, r>0$ and $\theta \in \mathbf{R}$. Setting $w:=z+r \mathrm{e}^{\mathrm{i} \theta}$ we conclude from (3.1) that

$$
\frac{1}{L}\left|\frac{H(w)-H(z)}{w-z}\right| \leq\left|\frac{H(w)-H(z)}{w-z}+\frac{\overline{w-z}}{w-z} \frac{\overline{G(w)-G(z)}}{\overline{w-z}}\right|,
$$

and letting $r$ tend to 0 we obtain

$$
\frac{1}{L}\left|H^{\prime}(z)\right| \leq\left|H^{\prime}(z)+\mathrm{e}^{-2 \mathrm{i} \theta} \overline{G^{\prime}(z)}\right| .
$$

Hence choosing suitably $\theta$ we have

$$
\frac{1}{L}\left|H^{\prime}(z)\right| \leq\left|H^{\prime}(z)\right|-\left|G^{\prime}(z)\right|
$$

and thus

$$
\left|G^{\prime}(z)\right| \leq\left(1-\frac{1}{L}\right)\left|H^{\prime}(z)\right|
$$

Combining this with $(0.2)$ we deduce that $L \geq 1$, and $\left|\mu_{F}(z)\right| \leq 1-\frac{1}{L}$. Since $z$ is an arbitrary point in $\mathbf{D}$, we see that $\left\|\mu_{F}\right\|_{1, \infty} \leq 1-\frac{1}{L}$, and thus $F$ is a quasiconformal mapping, which proves the lemma. 
Theorem 3.2. Given $\alpha \in[0 ; 1)$ suppose that $\Omega:=F(\mathbf{D})$ is an $\alpha$-convex domain and $\left\|\mu_{F}\right\|_{1, \infty}<1$. Then for every $k$ satisfying $\left\|\mu_{F}\right\|_{1, \infty} \leq k<1$ and for all $z_{1}, z_{2} \in \mathbf{D}$,

$$
\frac{\cos \frac{\alpha \pi}{2}-k}{\left(1-k^{2}\right) \cos \frac{\alpha \pi}{2}}\left|F\left(z_{2}\right)-F\left(z_{1}\right)\right| \leq\left|H\left(z_{2}\right)-H\left(z_{1}\right)\right| \leq \frac{\left|F\left(z_{2}\right)-F\left(z_{1}\right)\right|}{(1-k) \cos \frac{\alpha \pi}{2}},
$$

or equivalently, for such $k$ and all $w_{1}, w_{2} \in \Omega$,

$$
\frac{\cos \frac{\alpha \pi}{2}-k}{\left(1-k^{2}\right) \cos \frac{\alpha \pi}{2}}\left|w_{2}-w_{1}\right| \leq\left|H \circ F^{-1}\left(w_{2}\right)-H \circ F^{-1}\left(w_{1}\right)\right| \leq \frac{\left|w_{2}-w_{1}\right|}{(1-k) \cos \frac{\alpha \pi}{2}} .
$$

In consequence, if $\left\|\mu_{F}\right\|_{1, \infty}<\cos \frac{\alpha \pi}{2}$, then the function $H \circ F^{-1}$ is bi-Lipschitz.

Proof. Given $\alpha \in[0 ; 1)$ suppose that $\Omega$ is an $\alpha$-convex domain and $\left\|\mu_{F}\right\|_{1, \infty} \leq$ $k<1$. Fix $z_{1}, z_{2} \in \mathbf{D}$ such that $z_{1} \neq z_{2}$. From the first inequality in (2.2) it follows that

$$
\frac{\cos \frac{\alpha \pi}{2}-k}{\left(1-k^{2}\right) \cos \frac{\alpha \pi}{2}} \leq \operatorname{Re} \frac{H\left(z_{2}\right)-H\left(z_{1}\right)}{F\left(z_{2}\right)-F\left(z_{1}\right)} \leq\left|\frac{H\left(z_{2}\right)-H\left(z_{1}\right)}{F\left(z_{2}\right)-F\left(z_{1}\right)}\right|,
$$

which yields the first inequality in (3.2). The second inequality in (3.2) follows immediately from (2.5). The one-to-one correspondence $\mathbf{D} \ni z \mapsto w=F(z) \in \Omega$ shows that both the double inequalities (3.2) and (3.3) are equivalent. Finally, if $\left\|\mu_{F}\right\|_{1, \infty}<\cos \frac{\alpha \pi}{2}$, then the left term in (3.4) with $k:=\left\|\mu_{F}\right\|_{1, \infty}$ is positive. Hence and by (3.3) we conclude that $H \circ F^{-1}$ is a bi-Lipschitz mapping, and the proof is complete.

By Lemma 3.1 and Theorem 3.2 we easily obtain the following property.

Corollary 3.3. Suppose that $\Omega:=F(\mathbf{D})$ is an $\alpha$-convex domain for some $\alpha \in$ $[0 ; 1)$. Then $F$ is a quasiconformal mapping iff there exists a constant $L \geq 1$ satisfying (3.1) in Lemma 3.1.

Using the functions $S_{1}$ and $S_{2}$ given in (2.14) and (2.15), respectively, we define a function $S:\{(1,0)\} \cup\left\{(k, \alpha) \in[0 ; 1) \times[0 ; 1): k<\cos \frac{\alpha \pi}{2}\right\} \rightarrow \mathbf{R}$ by the following formulas: $S(1,0):=1$,

$$
S(k, 0):=S_{2}(k, 0)=k \cdot \sqrt{\frac{(1-k)^{2}+1}{(1-k)^{2}+k^{2}}} \quad \text { as } 0 \leq k<1,
$$

and for $\alpha \in(0 ; 1)$,

$$
S(k, \alpha):=\left\{\begin{array}{l}
S_{1}(k, \alpha) \quad \text { as } \tan \frac{(1-\alpha) \pi}{4}<k<\cos \frac{\alpha \pi}{2} \\
S_{2}(k, \alpha) \quad \text { as } 0 \leq k \leq \tan \frac{(1-\alpha) \pi}{4} .
\end{array}\right.
$$

Theorem 3.4. Given $\alpha \in[0 ; 1)$ and $k>0$ suppose that $\Omega:=F(\mathbf{D})$ is an $\alpha$-convex domain and $\left\|\mu_{F}\right\|_{1, \infty} \leq k<\cos \frac{\alpha \pi}{2}$. Then for every $a \in \mathbf{D}(1 / S(k, \alpha))$,

$$
\frac{(1-|a| S(k, \alpha))\left(\cos \frac{\alpha \pi}{2}-k\right)}{\left(1-k^{2}\right) \cos \frac{\alpha \pi}{2}}\left|F\left(z_{1}\right)-F\left(z_{2}\right)\right| \leq\left|F_{a}\left(z_{1}\right)-F_{a}\left(z_{2}\right)\right|, \quad z_{1}, z_{2} \in \mathbf{D} \text {. }
$$

In particular, $F_{a}$ is a quasiconformal mapping and

$$
\left\|\mu_{F_{a}}\right\|_{1, \infty} \leq k|a|<\frac{k}{S(k, \alpha)}<1 .
$$


If furthermore there exists a constant $L>0$ such that

$$
\frac{1}{L}\left|z_{1}-z_{2}\right| \leq\left|F\left(z_{1}\right)-F\left(z_{2}\right)\right|, \quad z_{1}, z_{2} \in \mathbf{D},
$$

then for every $a \in \mathbf{D}(1 / S(k, \alpha))$ the mapping $F_{a}$ is co-Lipschitz and

$$
\frac{(1-|a| S(k, \alpha))\left(\cos \frac{\alpha \pi}{2}-k\right)}{L\left(1-k^{2}\right) \cos \frac{\alpha \pi}{2}}\left|z_{1}-z_{2}\right| \leq\left|F_{a}\left(z_{1}\right)-F_{a}\left(z_{2}\right)\right|, \quad z_{1}, z_{2} \in \mathbf{D} .
$$

Proof. Given $\alpha \in[0 ; 1)$ suppose that $\Omega$ is an $\alpha$-convex domain. Fix $z_{1}, z_{2} \in \mathbf{D}$ such that $z_{1} \neq z_{2}$ and $a \in \mathbf{D}(1 / S(k, \alpha))$. From Lemma 2.4 and (3.6) it follows that

$$
\left|\frac{a\left(\bar{G}\left(z_{2}\right)-\bar{G}\left(z_{1}\right)\right)}{H\left(z_{2}\right)-H\left(z_{1}\right)}\right| \leq|a| S(k, \alpha)<1
$$

Hence

$$
\begin{aligned}
\left|F_{a}\left(z_{1}\right)-F_{a}\left(z_{2}\right)\right| & =\left|H\left(z_{1}\right)-H\left(z_{2}\right)\right|\left|1+\frac{a\left(\bar{G}\left(z_{2}\right)-\bar{G}\left(z_{1}\right)\right)}{H\left(z_{2}\right)-H\left(z_{1}\right)}\right| \\
& \geq\left|H\left(z_{1}\right)-H\left(z_{2}\right)\right|\left(1-\left|\frac{a\left(\bar{G}\left(z_{2}\right)-\bar{G}\left(z_{1}\right)\right)}{H\left(z_{2}\right)-H\left(z_{1}\right)}\right|\right) \\
& \geq\left|H\left(z_{1}\right)-H\left(z_{2}\right)\right|(1-|a| S(k, \alpha)) .
\end{aligned}
$$

Applying now Theorem 3.2 we obtain (3.7), and thus the mapping $F_{a}$ is injective.

By $(0.2)$, we see that $\partial F_{a}(z)=H^{\prime}(z) \neq 0$ as $z \in \mathbf{D}$. Then for every $z \in \mathbf{D}$,

$$
\left|\frac{\bar{\partial} F_{a}(z)}{\partial F_{a}(z)}\right|=\left|\frac{a \overline{G^{\prime}(z)}}{H^{\prime}(z)}\right|=|a|\left|\frac{\bar{\partial} F(z)}{\partial F(z)}\right| \leq|a|\left\|\mu_{F}\right\|_{1, \infty} \leq k|a|<\frac{k}{S(k, \alpha)} .
$$

Since $S_{1}(k, \alpha) \geq 1$ and $S_{2}(k, \alpha)>k$, we have $S(k, \alpha)>k$. Therefore (3.13) yields (3.8). Thus $F_{a}$ is a quasiconformal mapping.

If we assume additionally that (3.9) holds, then (3.7) together with (3.9) leads to (3.10), which means that $F_{a}$ is a co-Lipschitz mapping, and the proof is completed.

Corollary 3.5. Given $\alpha \in[0 ; 1)$ and positive numbers $k, L_{1}, L_{2}$ suppose that $\Omega$ is a bounded $\alpha$-convex domain, $\left(L_{1} L_{2}-1\right)\left(L_{1} L_{2}+1\right)^{-1} \leq k<\cos \frac{\alpha \pi}{2}$ and the function $F$ is bi-Lipschitz with constants $L_{1}, L_{2}$, i.e.

$$
\frac{1}{L_{1}}\left|z_{2}-z_{1}\right| \leq\left|F\left(z_{2}\right)-F\left(z_{1}\right)\right| \leq L_{2}\left|z_{2}-z_{1}\right|, \quad z_{1}, z_{2} \in \mathbf{D} \text {. }
$$

Then for every $a \in \mathbf{D}(1 / S(k, \alpha))$ the mapping $F_{a}$ is also bi-Lipschitz and the following inequality

$$
\begin{aligned}
\frac{(1-|a| S(k, \alpha))\left(\cos \frac{\alpha \pi}{2}-k\right)}{L_{1}\left(1-k^{2}\right) \cos \frac{\alpha \pi}{2}}\left|z_{1}-z_{2}\right| & \leq\left|F_{a}\left(z_{1}\right)-F_{a}\left(z_{2}\right)\right| \\
& \leq(1+|a| k) L_{2}\left|z_{1}-z_{2}\right|, \quad z_{1}, z_{2} \in \mathbf{D},
\end{aligned}
$$

holds. In particular, $F_{a}$ is a quasiconformal mapping and the estimation (3.8) holds.

Proof. Since $F$ is differentiable at an arbitrarily fixed point $\zeta_{0} \in \mathbf{D}$, the property (0.10) holds. Then for each $\theta \in \mathbf{R}$, we conclude from the first inequality in (3.14) 
that

$$
\begin{aligned}
\left|\partial F\left(\zeta_{0}\right) \mathrm{e}^{\mathrm{i} \theta}+\bar{\partial} F\left(\zeta_{0}\right) \mathrm{e}^{-\mathrm{i} \theta}\right| & =\frac{1}{r}\left|F\left(r \mathrm{e}^{\mathrm{i} \theta}+\zeta_{0}\right)-F\left(\zeta_{0}\right)\right|+\frac{1}{r}\left|\mathrm{o}\left(r \mathrm{e}^{\mathrm{i} \theta}\right)\right| \\
& \geq \frac{1}{L_{1}}+\frac{1}{r}\left|\mathrm{o}\left(r \mathrm{e}^{\mathrm{i} \theta}\right)\right| \rightarrow \frac{1}{L_{1}} \quad \text { as } r \rightarrow 0 .
\end{aligned}
$$

On the other hand side $\partial F\left(\zeta_{0}\right)=\left|\partial F\left(\zeta_{0}\right)\right| \mathrm{e}^{\mathrm{i} \alpha}$ and $\bar{\partial} F\left(\zeta_{0}\right)=\left|\bar{\partial} F\left(\zeta_{0}\right)\right| \mathrm{e}^{\mathrm{i} \beta}$ for some $\alpha, \beta \in \mathbf{R}$. Thus for $\theta:=(\pi+\beta-\alpha) / 2$ we obtain

$$
\left|\partial F\left(\zeta_{0}\right)\right|-\left|\bar{\partial} F\left(\zeta_{0}\right)\right| \geq \frac{1}{L_{1}}, \quad \zeta_{0} \in \mathbf{D} .
$$

From the second inequality in (3.14) it follows that the inequality (0.12) holds with $L$ replaced by $L_{2}$. Combining this with (3.16) we obtain

$$
\frac{|\partial F(z)|+|\bar{\partial} F(z)|}{|\partial F(z)|-|\bar{\partial} F(z)|} \leq L_{1} L_{2}, \quad z \in \mathbf{D} .
$$

Since $F$ is a sense-preserving diffeomorphic mapping, we deduce from (3.17) that $F$ is a $L_{1} L_{2}$-quasiconformal mapping, and consequently $\left\|\mu_{F}\right\|_{1, \infty} \leq k$. Then Theorem 3.4 and Proposition 0.1 imply the condition (3.15) for every $a \in \mathbf{D}(1 / S(k, \alpha))$, which means that $F_{a}$ is a bi-Lipschitz mapping. Moreover, Theorem 3.4 implies that $F_{a}$ is a quasiconformal mapping and the estimation (3.8) holds, which completes the proof.

Remark 3.6. All the results presented so far hold in particular in the case, where $\Omega$ is a convex domain, i.e. $\Omega$ is a 0 -convex domain. Then there is no restriction on the maximal dilatation of $F$, and so these results are valid for every quasiconformal harmonic mapping $F$. Furthermore, for $\alpha=0$ the function $S$ takes the simpler form (3.5). Hence the inequalities (3.10) and (3.8) can be simplified as follows

$$
\frac{1-|a| k \cdot \sqrt{\frac{(1-k)^{2}+1}{(1-k)^{2}+k^{2}}}}{L(1+k)}\left|z_{1}-z_{2}\right| \leq\left|F_{a}\left(z_{1}\right)-F_{a}\left(z_{2}\right)\right|, \quad z_{1}, z_{2} \in \mathbf{D},
$$

and

$$
\left\|\mu_{F_{a}}\right\|_{1, \infty} \leq k|a|<\sqrt{\frac{(1-k)^{2}+k^{2}}{(1-k)^{2}+1}}<1
$$

provided

$$
k>0 \text { and }|a|<\frac{1}{k} \cdot \sqrt{\frac{(1-k)^{2}+k^{2}}{(1-k)^{2}+1}} .
$$

This extends Kalaj's Theorem A in the case where $F$ is a quasiconformal mapping.

In the case where $F(\mathbf{D})$ is a convex domain we obtain the following Lipschitz and co-Lipchitz properties of the mapping $F_{a} \circ H^{-1}$.

Theorem 3.7. Suppose that $\Omega:=F(\mathbf{D})$ is a convex domain and that $a \in \mathbf{C}$ satisfies $|a| \leq 1$ (resp. $\left.|a|<1 / S\left(\left\|\mu_{F}\right\|_{1, \infty}, 0\right)\right)$ provided $\left\|\mu_{F}\right\|_{1, \infty}=1$ (resp. $\left\|\mu_{F}\right\|_{1, \infty}<$ 
1). Then $F_{a}:=H+a \bar{G}$ is an injective mapping, $F_{a} \circ H^{-1}$ is a Lipschitz mapping and

$$
\begin{aligned}
\left|H\left(z_{1}\right)-H\left(z_{2}\right)\right| & \geq \frac{1}{1+|a| S\left(\left\|\mu_{F}\right\|_{1, \infty}, 0\right)}\left|F_{a}\left(z_{1}\right)-F_{a}\left(z_{2}\right)\right| \\
& \geq \frac{1}{2}\left|F_{a}\left(z_{1}\right)-F_{a}\left(z_{2}\right)\right|, \quad z_{1}, z_{2} \in \mathbf{D} .
\end{aligned}
$$

Moreover, if $a \in \mathbf{C}$ satisfies $|a|<1 / S\left(\left\|\mu_{F}\right\|_{1, \infty}, 0\right)$, then $F_{a} \circ H^{-1}$ is a bi-Lipschitz mapping and

$$
\left|H\left(z_{1}\right)-H\left(z_{2}\right)\right| \leq \frac{1}{1-|a| S\left(\left\|\mu_{F}\right\|_{1, \infty}, 0\right)}\left|F_{a}\left(z_{1}\right)-F_{a}\left(z_{2}\right)\right|, \quad z_{1}, z_{2} \in \mathbf{D} .
$$

Proof. Suppose first that $\left\|\mu_{F}\right\|_{1, \infty}=1$ and $|a| \leq 1$. Then from (2.11) it follows that for all $z_{1}, z_{2} \in \mathbf{D}, z_{1} \neq z_{2}$,

$$
\begin{aligned}
\left|F_{a}\left(z_{1}\right)-F_{a}\left(z_{2}\right)\right| & =\left|H\left(z_{1}\right)-H\left(z_{2}\right)+a\left(\overline{G\left(z_{1}\right)}-\overline{G\left(z_{2}\right)}\right)\right| \\
& \geq\left|H\left(z_{1}\right)-H\left(z_{2}\right)\right|-|a|\left|G\left(z_{1}\right)-G\left(z_{2}\right)\right| \\
& \geq\left|H\left(z_{1}\right)-H\left(z_{2}\right)\right|-\left|G\left(z_{1}\right)-G\left(z_{2}\right)\right|>0,
\end{aligned}
$$

and similarly,

$$
\begin{aligned}
\left|F_{a}\left(z_{1}\right)-F_{a}\left(z_{2}\right)\right| & \leq\left|H\left(z_{1}\right)-H\left(z_{2}\right)\right|+|a|\left|G\left(z_{1}\right)-G\left(z_{2}\right)\right| \\
& \leq(1+|a|)\left|H\left(z_{1}\right)-H\left(z_{2}\right)\right| \leq 2\left|H\left(z_{1}\right)-H\left(z_{2}\right)\right| .
\end{aligned}
$$

Thus the mapping $F_{a}$ is injective and (3.21) holds. Furthermore, if $|a|<1=$ $1 / S(1,0)$, then the first inequality in $(3.23)$ and $(2.11)$ yield

$$
\left|F_{a}\left(z_{1}\right)-F_{a}\left(z_{2}\right)\right| \geq(1-|a|)\left|H\left(z_{1}\right)-H\left(z_{2}\right)\right|, \quad z_{1}, z_{2} \in \mathbf{D},
$$

which implies (3.22).

Suppose now that $\left\|\mu_{F}\right\|_{1, \infty}<1$ and $|a|<1 / S\left(\left\|\mu_{F}\right\|_{1, \infty}, 0\right)$. Then by Theorem 3.4 the mapping $F_{a}$ is quasiconformal and (3.12) with $k:=\left\|\mu_{F}\right\|_{1, \infty}$ implies (3.22). Modifying suitably (3.12) we conclude from Lemma 2.4 and (3.5) that

$$
\begin{aligned}
\left|F_{a}\left(z_{1}\right)-F_{a}\left(z_{2}\right)\right| & \leq\left|H\left(z_{1}\right)-H\left(z_{2}\right)\right|\left(1+\left|\frac{a\left(\overline{G\left(z_{1}\right)}-\overline{G\left(z_{2}\right)}\right)}{H\left(z_{1}\right)-H\left(z_{2}\right)}\right|\right) \\
& \leq\left(1+|a| S\left(\left\|\mu_{F}\right\|_{1, \infty}, 0\right)\right)\left|H\left(z_{1}\right)-H\left(z_{2}\right)\right| \\
& \leq 2\left|H\left(z_{1}\right)-H\left(z_{2}\right)\right|, \quad z_{1}, z_{2} \in \mathbf{D},
\end{aligned}
$$

which shows (3.21).

Finally in the case where $F(\mathbf{D})$ is a convex domain we can give several sufficient and necessary conditions for $F$ to be quasiconformal.

Theorem 3.8. Suppose that $\Omega:=F(\mathbf{D})$ is a convex domain. Then the following five conditions are equivalent to each other:

(i) $F$ is a quasiconformal mapping;

(ii) there exists a constant $L_{1}$ such that $1 \leq L_{1}<2$ and

$$
\left|F\left(z_{2}\right)-F\left(z_{1}\right)\right| \leq L_{1}\left|H\left(z_{2}\right)-H\left(z_{1}\right)\right|, \quad z_{1}, z_{2} \in \mathbf{D} ;
$$

(iii) there exists a constant $l_{1}$ such that $0 \leq l_{1}<1$ and

$$
\left|G\left(z_{2}\right)-G\left(z_{1}\right)\right| \leq l_{1}\left|H\left(z_{2}\right)-H\left(z_{1}\right)\right|, \quad z_{1}, z_{2} \in \mathbf{D} ;
$$


(iv) there exists a constant $L_{2} \geq 1$ such that

$$
\left|H\left(z_{2}\right)-H\left(z_{1}\right)\right| \leq L_{2}\left|F\left(z_{2}\right)-F\left(z_{1}\right)\right|, \quad z_{1}, z_{2} \in \mathbf{D} ;
$$

(v) $H \circ F^{-1}$ and $F \circ H^{-1}$ are bi-Lipschtz mappings.

Moreover, the following implications hold: (3.24) $\Longrightarrow\left\|\mu_{F}\right\|_{1, \infty} \leq L_{1}-1,(3.25) \Longrightarrow$ $\left\|\mu_{F}\right\|_{1, \infty} \leq l_{1}$ and $(3.26) \Longrightarrow\left\|\mu_{F}\right\|_{1, \infty} \leq 1-\frac{1}{L_{2}}$.

Proof. Since

$$
\left|F\left(z_{2}\right)-F\left(z_{1}\right)\right| \leq\left|H\left(z_{2}\right)-H\left(z_{1}\right)\right|+\left|G\left(z_{2}\right)-G\left(z_{1}\right)\right|, \quad z_{1}, z_{2} \in \mathbf{D},
$$

we see that (iii) implies (ii). In the same way as in the proof of Lemma 3.1 we deduce that (3.24) implies that for every $z \in \mathbf{D}$,

$$
\left|H^{\prime}(z)\right|+\left|G^{\prime}(z)\right| \leq L_{1}\left|H^{\prime}(z)\right|,
$$

and so $\left|G^{\prime}(z)\right| /\left|H^{\prime}(z)\right| \leq L_{1}-1$. Hence (ii) implies (i) and $\left\|\mu_{F}\right\|_{1, \infty} \leq L_{1}-1$. By Lemma 2.4, (i) implies (iii) and it is easily seen that (3.25) yields $\left\|\mu_{F}\right\|_{1, \infty} \leq l_{1}$. From Lemma 3.1 we conclude that (iv) implies (i) and $\left\|\mu_{F}\right\|_{1, \infty} \leq 1-\frac{1}{L_{2}}$. Theorem 3.2 shows that (i) implies (v). Finally, (v) clearly implies (iv), and the proof is complete.

\section{Complementary results}

The results presented so far involve the $\alpha$-convexity of the range domain $F(\mathbf{D})$ with the maximal dilatation $K$ of a quasiconformal harmonic mapping $F$ in $\mathbf{D}$. Obviously, each convex domain is a 0 -convex domain. Therefore the concept of $\alpha$-convexity remarkably extends the convexity property, and so our results are applicable for a wide family of quasiconformal harmonic mappings. However, the detailed discussion exceeds the scope of this paper. Therefore here we confine ourselves only to the case where $F$ is a quasi-conformal harmonic self-mapping of $\mathbf{D}$. Then the bi-Lipschitz behaviour of $F$ follows from the result of Pavlović; cf. [12, Thm. 1.2]. Moreover, if $F(0)=0$ and $F$ is a $K$-quasiconformal mapping, then the more precise result [11, Thm. 3.3] asserts that for all $z, w \in \mathbf{D}$ :

$$
\frac{L_{K}^{3 K}}{K^{4 K+1} M_{K}^{K}}|z-w| \leq|F(z)-F(w)| \leq K\left(M_{K} K\right)^{K}|z-w|,
$$

where for every $t \geq 1$,

$$
M_{t}:=\frac{4}{\pi} \int_{0}^{1 / \sqrt{2}}\left(\frac{\Phi_{t}(r)}{r}\right)^{1+1 / t} \frac{\mathrm{d} r}{\sqrt{1-r^{2}}}
$$

and

$$
L_{t}:=\frac{4}{\pi} \int_{0}^{1 / \sqrt{2}}\left(\frac{\Phi_{1 / t}(r)}{r}\right)^{1+1 / t} \frac{\mathrm{d} r}{\sqrt{1-r^{2}}} .
$$

Here $\Phi_{t}$ denotes the Hersch-Pfluger distortion function defined for each $t>0$ by the equalities

$$
\Phi_{t}(r):=\mu^{-1}(\mu(r) / t), \quad 0<r<1 ; \quad \Phi_{t}(0):=0, \Phi_{t}(1):=1,
$$

where $\mu$ stands for the module of the Grötzsch extremal domain $\mathbf{D} \backslash[0 ; r]$; cf. [4] and [6, pp. 53 and 63]. For many useful properties of the Hersch-Pfluger distortion 
function the reader is referred to [1]. Efficient methods for the approximation of this function are discussed in [9], [8], [13] and [14].

If we consider the maximal dilatation $K:=\left(1+\left\|\mu_{F}\right\|_{1, \infty}\right) /\left(1-\left\|\mu_{F}\right\|_{1, \infty}\right)$ of $F$ instead of $k:=\left\|\mu_{F}\right\|_{1, \infty}$, it is more convenient to use the function $S^{*}$ defined on the set $\left\{(K, \alpha) \in[1 ;+\infty) \times[0 ; 1): \frac{K-1}{K+1}<\cos \frac{\alpha \pi}{2}\right\}$ by

$$
S^{*}(K, \alpha):=S\left(\frac{K-1}{K+1}, \alpha\right)
$$

as compared to $S$. By (3.5) we have

$$
S^{*}(K, 0)=\frac{K-1}{K+1} \cdot \sqrt{\frac{(K+1)^{2}+4}{(K-1)^{2}+4}}, \quad K \geq 1 .
$$

Theorem 4.1. Given $K>1$ suppose that $F$ is a $K$-quasiconformal harmonic self-mapping of $\mathbf{D}$ normalized by $F(0)=0$. Then for every $a \in \mathbf{D}\left(1 / S^{*}(K, 0)\right), F_{a}$ is a bi-Lipschitz mapping satisfying

$$
\begin{aligned}
& \frac{\left(1-|a| S^{*}(K, 0)\right)(K+1)}{2 K} \frac{L_{K}^{3 K}}{K^{4 K+1} M_{K}^{K}}\left|z_{1}-z_{2}\right| \leq\left|F_{a}\left(z_{1}\right)-F_{a}\left(z_{2}\right)\right| \\
& \leq\left(1+|a| \frac{K-1}{K+1}\right) K\left(M_{K} K\right)^{K}\left|z_{1}-z_{2}\right|, \quad z_{1}, z_{2} \in \mathbf{D} .
\end{aligned}
$$

In particular, $F_{a}$ is a $K^{*}$-quasiconformal mapping with

$$
K^{*} \leq \frac{K+1+(K-1)|a|}{K+1-(K-1)|a|}<\frac{(K+1) S^{*}(K, 0)+(K-1)}{(K+1) S^{*}(K, 0)-(K-1)} .
$$

Proof. Obviously, $\mathbf{D}$ is a convex domain, and hence a 0 -convex domain. By the assumption, $\left\|\mu_{F}\right\|_{1, \infty} \leq k:=(K-1) /(K+1)<1=\cos (0)$ and $k>0$. From (4.5) we have $S^{*}(K, 0)=S(k, 0)$. Then the first inequality in (4.7) follows directly from Theorem 3.4 and the first inequality in (4.1). The second inequality in (4.7) is a direct conclusion from Proposition 0.1 and the second inequality in (4.1). The first inequality in (4.8) follows immediately from that in (3.8) of Theorem 3.4. We derive the second inequality in (4.8) from the assumption $|a|<1 / S^{*}(K, 0)$.

Remark 4.2. By [11, Lemma 1.3], the following estimations hold:

$$
\frac{K 2^{5\left(1-K^{2}\right) /(2 K)}}{K^{2}+K-1} \leq L_{K} \leq 1 \leq M_{K} \leq K^{2} 2^{5\left(1-1 / K^{2}\right) / 2}, \quad K \geq 1 .
$$

Applying them we weaken the inequalities (4.7) to the following more explicit forms

$$
\begin{aligned}
& \frac{\left(1-|a| S^{*}(K, 0)\right)(K+1)}{2 K} \frac{2^{5\left(1-K^{2}\right)(3+1 / K) / 2}}{K^{3 K+1}\left(K^{2}+K-1\right)^{3 K}}\left|z_{1}-z_{2}\right| \leq\left|F_{a}\left(z_{1}\right)-F_{a}\left(z_{2}\right)\right| \\
& \leq\left(1+|a| \frac{K-1}{K+1}\right) K^{3 K+1} 2^{5(K-1 / K) / 2}\left|z_{1}-z_{2}\right|, \quad z_{1}, z_{2} \in \mathbf{D} .
\end{aligned}
$$

Corollary 4.3. If $K \geq 1$ and $F$ is a $K$-quasiconformal harmonic self-mapping of $\mathbf{D}$, then $H$ is a bi-Lipschitz mapping. If additionally $F$ is normalized by $F(0)=0$, then

$$
\begin{aligned}
\frac{K+1}{2 K} \frac{L_{K}^{3 K}}{K^{4 K+1} M_{K}^{K}}\left|z_{1}-z_{2}\right| & \leq\left|H\left(z_{1}\right)-H\left(z_{2}\right)\right| \\
& \leq K\left(M_{K} K\right)^{K}\left|z_{1}-z_{2}\right|, \quad z_{1}, z_{2} \in \mathbf{D} .
\end{aligned}
$$


Proof. Suppose first that $F$ is a $K$-quasiconformal mapping normalized by $F(0)=$ 0 . Since $H=F_{0}$, we conclude from Theorem 4.1 that $H$ is a bi-Lipschitz mapping and the inequalities (4.10) hold. In the case where $F(0) \neq 0$ we can see that $F \circ \eta(0)=0$ for certain conformal self-mapping $\eta$ of $\mathbf{D}$. Then $F \circ \eta$ is a bi-Lipschitz mapping, and so is $F$.

As an application of Corollary 4.3 we derive the following necessary and sufficient condition for quasiconformality of a harmonic self-mapping of $\mathbf{D}$.

Corollary 4.4. For every harmonic and injective self-mapping $F$ of $\mathbf{D}, F$ is quasiconformal iff $H$ is a Lipschitz mapping and

$$
\inf _{z \in \mathbf{D}} \mathrm{J}[F](z)>0 .
$$

Proof. Suppose that $F$ is a quasiconformal mapping. Then Corollary 4.3 shows that $H$ is a Lipschitz mapping. On the other hand side, by [10, Thm. 3.2], the condition (4.11) holds.

Conversely, suppose now that $H$ is a Lipschitz mapping and the condition (4.11) holds. Then there exist some positive constans $C_{1}$ and $C_{2}$ satisfying

$$
0<C_{1} \leq \mathrm{J}[F](z)=\left|H^{\prime}(z)\right|^{2}-\left|G^{\prime}(z)\right|^{2} \quad \text { and } \quad\left|H^{\prime}(z)\right| \leq C_{2}<+\infty, \quad z \in \mathbf{D} .
$$

Hence for every $z \in \mathbf{D}$,

$$
\left|\frac{\bar{\partial} F(z)}{\partial F(z)}\right|^{2}=\frac{\left|G^{\prime}(z)\right|^{2}}{\left|H^{\prime}(z)\right|^{2}} \leq \frac{\left|H^{\prime}(z)\right|^{2}-C_{1}}{\left|H^{\prime}(z)\right|^{2}}=1-\frac{C_{1}}{\left|H^{\prime}(z)\right|^{2}} \leq 1-\frac{C_{1}}{C_{2}^{2}}<1,
$$

and consequently $F$ is a quasiconformal mapping, which completes the proof.

\section{References}

[1] Anderson, G. D., M. K. Vamanamurthy, and M. Vuorinen: Distortion functions for plane quasiconformal mappings. - Israel J. Math. 62, 1988, 1-16.

[2] Chunqui, M., and R. Hernández: Univalent harmonic mappings and linearly connected domains. - J. Math. Anal. Appl. 332, 2007, 1189-1194.

[3] Clunie, J., and T. Sheil-Small: Harmonic univalent functions. - Ann. Acad. Sci. Fenn. Ser. A I Math. 9, 1984, 3-25.

[4] Hersch, J., and A. Pfluger: Généralisation du lemme de Schwarz et du principe de la mesure harmonique pour les fonctions pseudo-analytiques. - C. R. Math. Acad. Sci. Paris 234, 1952, 43-45.

[5] KalaJ, D.: Quasiconformal harmonic mappings and close to convex domains. - Filomat 24:1, 2010, 63-68.

[6] Lehto, O., and K. I. Virtanen: Quasiconformal mappings in the plane. - Grundlehren Math. Wiss. 126, 2nd edition, Springer, Berlin, 1973.

[7] Lewy, H.: On the non-vanishing of the Jacobian in certain one-to-one mappings. - Bull. Amer. Math. Soc. 42, 1936, 689-692.

[8] PARTyKA, D.: Approximation of the Hersch-Pfluger distortion function. Applications. - Ann. Univ. Mariae Curie-Skłodowska Sect. A 45, 1992, 99-111.

[9] Partyka, D.: Approximation of the Hersch-Pfluger distortion function. - Ann. Acad. Sci. Fenn. Ser. A I Math. 18, 1993, 343-354.

[10] PARTYKA, D., and K. SAKAn: Distortion of the area measure for one-to-one harmonic mappings of the unit disk onto itself. - Sci. Bull. of Chełm, Sect. of Math. and Comp. Sci. 2, 2007, 39-48. 
[11] Partyka, D., and K. SAKan: On bi-Lipschitz type inequalities for quasiconformal harmonic mappings. - Ann. Acad. Sci. Fenn. Math. 32, 2007, 579-594.

[12] Pavlović, M.: Boundary correspondence under harmonic quasiconformal homeomorphisms of the unit disk. - Ann. Acad. Sci. Fenn. Math. 27, 2002, 365-372.

[13] ZAJA̧C, J.: Functional identities for special functions of quasiconformal theory. - Ann. Acad. Sci. Fenn. Ser. A I Math. 18, 1993, 93-103.

[14] ZAJA̧C, J.: Quasihomographies in the theory of Teichmüller spaces. - Dissertationes Math. 357, Institute of Mathematics, Polish Academy of Sciences, Warszawa, 1996.

Received 5 December 2011 